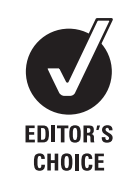

See Editorial, p 185

- A supplementary table is published online only at http:// thorax.bmj.com/content/vol64/ issue3

${ }^{1}$ Institute of Clinical Medicine, National Yang-Ming University, Taipei, Taiwan; ${ }^{2}$ Department of Surgery, Cathay General Hospital and School of Medicine, Fu Jen Catholic University, Taipei,

Taiwan; ${ }^{3}$ Division of Thoracic

Surgery, Department of Surgery, Taipei Veterans General Hospital and School of Medicine,

National Yang-Ming University,

Taipei, Taiwan

Correspondence to:

Dr Y-C Wu, Division of Thoracic Surgery, Department of Surgery, Taipei Veterans General Hospital, No 201, Section 2,

Shih-Pai Road, Taipei 112,

Taiwan; wuyc@vghtpe.gov.tw

Received 18 December 2007 Accepted 24 August 2008

\title{
Post-recurrence survival in completely resected stage I non-small cell lung cancer with local recurrence
}

\author{
J-J Hung, ${ }^{1,2,3}$ W-H Hsu, ${ }^{3}$ C-C Hsieh, ${ }^{3}$ B-S Huang, ${ }^{3}$ M-H Huang, ${ }^{3}$ J-S Liu, ${ }^{2}$ Y-C Wu ${ }^{3}$
}

\begin{abstract}
Objective: Resection is the best treatment for patients with stage I non-small cell lung cancer (NSCLC). Patterns of disease recurrence after complete resection in stage I NSCLC have not been well demonstrated. The aim of this study was to evaluate the prognostic predictors of postrecurrence survival in patients with resected stage I NSCLC with local recurrence.
\end{abstract}

Methods: The clinicopathological characteristics of 123 patients with local recurrence after complete resection of stage I NSCLC in Taipei Veterans General Hospital between 1980 and 2000 were retrospectively reviewed. Post-recurrence survival and their predictors were analysed.

Results: The patterns of local recurrence included local only in $74(60.2 \%)$ and both local and distant in 49 (39.8\%) patients. The 1 and 2 year post-recurrence survival rates for the 74 patients with local only recurrence were $48.7 \%$ and $17.6 \%$, respectively. Tumour size $(p=0.033)$ and treatment for initial recurrence $(p<0.001)$ were significant predictors for post-recurrence survival in 74 patients with local only recurrence in univariate analyses. The hazard of death was greater in patients with larger tumour size. Treatment for initial recurrence $(p=0.001)$ was still a significant prognostic indicator in multivariate analyses. Patients who underwent reoperation after local recurrence survived longer than those who received chemotherapy and/or radiotherapy and those that received no treatment.

Conclusions: Treatment for initial recurrence is a prognostic predictor for post-recurrence survival in resected stage I NSCLC with local recurrence. Complete surgical resection should be considered in selected candidates with resectable local recurrent disease.

Lung cancer is the leading cause of cancer death worldwide. Surgical resection is the treatment of choice for early stage non-small cell lung cancer (NSCLC). ${ }^{1}$ Patients with stage I disease have the most favourable prognosis. Five year survival after resection ranges between $55 \%$ and $80 \% .^{1-5}$ Recurrence is the most common cause of disease failure and mortality for NSCLC after resection..$^{4-8}$ Reported recurrence rates after surgical resection in stage I NSCLC range from $27 \%$ to $38 \%{ }^{4-6}$ The incidence of local or regional recurrence in stage I NSCLC after surgical resection has been reported to be between $7 \%$ and $15 \%{ }^{46}$ Chemotherapy, radiotherapy or combined therapy are common treatment modalities for patients with resected NSCLC after recurrence. However, reoperation after recurrence is only appropriate in a few patients. ${ }^{7-9}$
Although the prognosis of stage I NSCLC is relatively good compared with more advanced stages, ${ }^{3-5}$ the course and predictors of post-recurrence survival in resected stage I NSCLC with local recurrence have rarely been reported. Few studies have focused on the outcome and post-recurrence survival of surgically treated patients with stage I NSCLC after local recurrence. In this report, we analysed the patterns of local recurrence and demonstrated the prognostic predictors of postrecurrence survival in resected stage I NSCLC with local recurrence.

\section{PATIENTS AND METHODS}

Between January 1980 and December 2000, 970 patients underwent resections for pathological stage I NSCLC at Taipei Veterans General Hospital. The preoperative staging workup was done as previously described. ${ }^{10}{ }^{11}$ Chest and upper abdomen CT scans, nuclear medicine survey (bone and brain) and bronchoscopy were performed before operation. Mediastinoscopy was performed only when the CT scan showed enlarged mediastinal lymph nodes (diameter $>1.0 \mathrm{~cm}$ ). Patients suspected of having distant metastases were excluded from consideration. All patients underwent complete resection of the lung cancer with mediastinal lymph node dissection as previously described. ${ }^{10} 11$ The resected specimens and all dissected regional nodes were examined for pathological staging. None of the patients had received adjuvant therapy after initial surgical resection for stage I NSCLC. The hospital charts of all patients were reviewed, including operative records and pathological reports. Histological typing was determined according to the World Health Organization classification. ${ }^{12}$ Determination of disease stages was based on the TNM classification of the International Union Against Cancer. ${ }^{13}$

All patients were followed-up at our outpatient department with an interval of 3 months in the first 2 years after resection and an interval of 6 months thereafter. Of the 970 patients, complete follow-up was available in 933 patients (96.2\%). During that period, local recurrence after surgical resection was detected in $123(13.2 \%)$ of the 933 patients. The characteristics of these 123 patients according to patterns of local recurrence (local only or local with distant) are listed in table 1. To investigate their impact on post-recurrence survival, the following clinicopathological factors were used in univariate and multivariate analyses: age at recurrence, gender, smoking index, laterality of tumour (right vs left), histological type of the 
Table 1 Characteristics of the 123 patients with resected stage I non-small cell lung cancer with local recurrence

\begin{tabular}{|c|c|c|}
\hline \multirow[b]{3}{*}{ Variables } & \multicolumn{2}{|c|}{ Pattern of local recurrence } \\
\hline & \multirow{2}{*}{$\begin{array}{l}\text { Local only } \\
\text { No of patients (\%) }\end{array}$} & \multirow{2}{*}{$\begin{array}{l}\text { Local and distant } \\
\text { No of patients (\%) }\end{array}$} \\
\hline & & \\
\hline Total No of patients & $74(60.2)$ & $49(39.8)$ \\
\hline Age at recurrence (years) (mean (SD)) & $64.5(9.9)$ & $65.5(7.9)$ \\
\hline \multicolumn{3}{|l|}{ Sex } \\
\hline Male & $65(87.8)$ & $41(83.7)$ \\
\hline Female & $9(12.2)$ & $8(16.3)$ \\
\hline Smoking index (pack-years) (median (IOR)) & $30.0(16.3-40.0)$ & $30.0(10.8-40.0)$ \\
\hline \multicolumn{3}{|l|}{ Tumour location } \\
\hline Right lung & $51(68.9)$ & $41(83.7)$ \\
\hline Left lung & $23(31.1)$ & $8(16.3)$ \\
\hline Tumour size $(\mathrm{cm})$ (mean (SD)) & $4.1(2.2)$ & $4.4(2.3)$ \\
\hline \multicolumn{3}{|l|}{ Histological type } \\
\hline Squamous cell carcinoma & $41(55.4)$ & $19(38.8)$ \\
\hline Adenocarcinoma & $24(32.4)$ & $21(42.8)$ \\
\hline Bronchioalveolar carcinoma & $6(8.1)$ & $4(8.2)$ \\
\hline Large cell carcinoma & $3(4.1)$ & $4(8.2)$ \\
\hline Adenosquamous carcinoma & $0(0.0)$ & $1(2.0)$ \\
\hline \multicolumn{3}{|l|}{ Extent of pulmonary resection } \\
\hline Sublobar resection & $10(13.5)$ & $1(2.0)$ \\
\hline Lobectomy or bilobectomy & $54(73.0)$ & $45(91.8)$ \\
\hline Pneumonectomy & $10(13.5)$ & $3(6.2)$ \\
\hline \multicolumn{3}{|l|}{ T status } \\
\hline $\mathrm{T} 1$ & $27(36.5)$ & $11(22.4)$ \\
\hline $\mathrm{T} 2$ & $47(63.5)$ & $38(77.6)$ \\
\hline \multicolumn{3}{|l|}{ Visceral pleural invasion } \\
\hline Absent & $56(75.7)$ & $44(89.8)$ \\
\hline Present & $15(20.2)$ & $4(8.2)$ \\
\hline Unknown & $3(4.1)$ & $1(2.0)$ \\
\hline No of LNs dissected (mean (SD)) & $12.0(8.3)$ & $16.1(12.4)$ \\
\hline $\mathrm{LN} \leqslant 15$ & $51(68.9)$ & $27(55.1)$ \\
\hline $\mathrm{LN}>15$ & $20(27.0)$ & $21(42.9)$ \\
\hline Unknown & $3(4.1)$ & $1(2.0)$ \\
\hline Disease free interval (months) (mean (SD)) & $18.4(12.0)$ & $18.0(15.6)$ \\
\hline$\leqslant 18$ & $40(54.1)$ & $30(61.2)$ \\
\hline$>18$ & $34(45.9)$ & $19(38.8)$ \\
\hline \multicolumn{3}{|l|}{ Mediastinal recurrence } \\
\hline Absent & $38(51.4)$ & $32(65.3)$ \\
\hline Present & $18(24.3)$ & $12(24.5)$ \\
\hline Unknown & $18(24.3)$ & $5(10.2)$ \\
\hline \multicolumn{3}{|l|}{ Treatment for recurrence } \\
\hline None & $7(9.5)$ & $9(18.4)$ \\
\hline With surgery & $8(10.8)$ & $2(4.1)$ \\
\hline Surgery only & 6 & 0 \\
\hline Surgery $+\mathrm{C} / \mathrm{T}$ and/or $\mathrm{R} / \mathrm{T}$ & 2 & 2 \\
\hline $\mathrm{C} / \mathrm{T}$ and/or $\mathrm{R} / \mathrm{T}$ & $26(35.1)$ & $27(55.1)$ \\
\hline $\mathrm{C} / \mathrm{T}$ only & 9 & 9 \\
\hline $\mathrm{R} / \mathrm{T}$ only & 11 & 9 \\
\hline $\mathrm{C} / \mathrm{T}+\mathrm{R} / \mathrm{T}$ & 6 & 9 \\
\hline Unknown & $33(44.6)$ & $11(22.4)$ \\
\hline
\end{tabular}

$\mathrm{C} / \mathrm{T}$, chemotherapy; IQR, interquartile range; $\mathrm{LN}$, lymph node; $\mathrm{R} / \mathrm{T}$, radiotherapy.

tumour (squamous cell carcinoma vs others), tumour size, extent of pulmonary resection (sublobar resection vs lobectomy or bilobectomy or pneumonectomy), $\mathrm{T}$ status (T2 vs T1), visceral pleural invasion (present vs absent), number of mediastinal lymph nodes dissected $(>15$ vs $\leqslant 15)$, disease free interval ( $>18$ vs $\leqslant 18$ months), mediastinal recurrence (present vs absent) and treatment for initial recurrence (none vs with surgery vs chemotherapy and/or radiotherapy). Secondary primary lung cancer was differentiated from recurrent NSCLC according to the criteria proposed by Martini and Melamed. ${ }^{4}{ }^{14}$ Local recurrence was defined as tumour recurrence in a contiguously anatomical site, including the ipsilateral hemithorax and mediastinum, after surgical resection. Distant recurrence was defined as tumour recurrence in the contralateral lung or outside the hemithorax and mediastinum after surgical resection. The length of post-recurrence survival was defined as the interval (in months) between the date of initial recurrence identified and the date of either death or the last follow-up. 


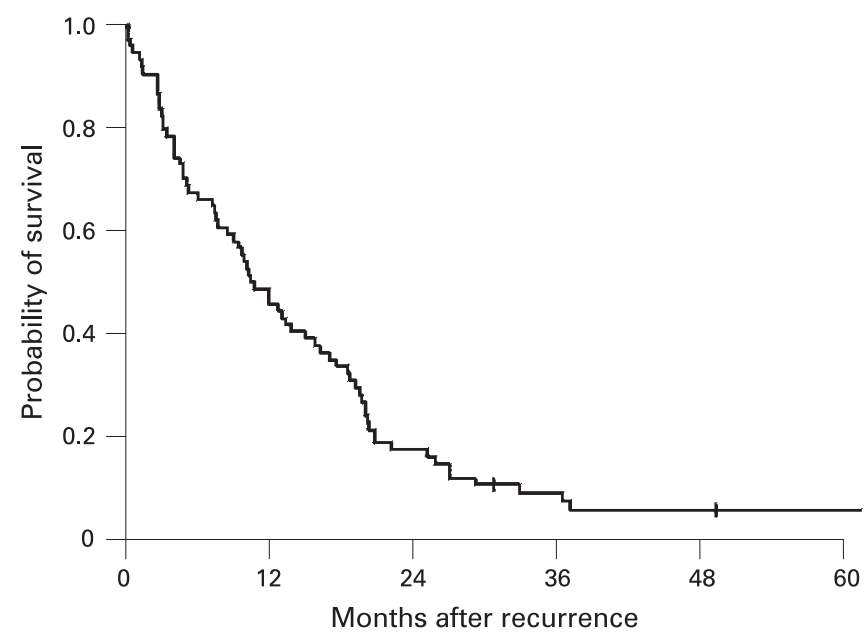

Figure 1 Cumulative probability of post-recurrence survival in 74 patients with resected stage I non-small cell lung cancer with local only recurrence.

Treatment for local recurrence included surgical resection, chemotherapy, radiotherapy and combined modalities.

Post-recurrence survival was calculated by the Kaplan-Meier method. ${ }^{15}$ Univariate and multivariate analyses were performed by means of the Cox proportional hazards model using SPSS software (V.12.0; SPSS, Chicago, Illinois, USA). Backward stepwise regression procedure was used. Data for treatment of recurrence were only available in 41 of the 74 patients with local only recurrence. Only these 41 patients were entered into the multivariate analyses. Statistical analysis was considered to be significant when the probability value was $<0.05$.

\section{RESULTS}

Median follow-up time for all 933 surgically resected stage I NSCLC patients was 60.7 months (mean 71.1 (57.1) months). The overall 5 and 10 year survival rates were $51.7 \%$ and $31.8 \%$, respectively. No recurrence was found in $644(69.0 \%)$ of the 933 patients. The patterns of recurrence included distant only in 166 $(17.8 \%)$, local only in $74(7.9 \%)$, and local and distant in 49 (5.3\%) patients.

Local recurrence after surgical resection developed in 123 (13.2\%) of the 933 patients. Median follow-up time for these 123 surgically resected stage I NSCLC patients with local recurrence was 28.9 months (mean 35.7 (26.0); range 5.4-204.8). Median time to initial recurrence for these 123 patients was
15.2 months (mean 18.3 (13.5)). The 1 and 2 year postrecurrence survival rates were $48.0 \%$ and $18.7 \%$, respectively. The patterns of local recurrence included local only in 74 $(60.2 \%)$ and both local and distant in $49(39.8 \%)$ patients (local with distant in 19, local before distant in 24 and distant before local in six). At the last follow-up session, only three patients were alive, 117 patients had died of cancer, one patient had died of other causes and two patients had died of unknown causes. Treatments after initial recurrence included surgery for 10 patients, chemotherapy for 18 patients, radiation for 20 patients and a combination of chemotherapy and radiotherapy for 15 patients. Sixteen patients had no treatment after initial recurrence (eight patients because of poor performance status, two patients sought alternative treatment, three patients refused treatment, one patient underwent surgery but the lesion was found to be unresectable and another two patients had unknown causes).

Median follow-up time for the 74 patients with resected stage I NSCLC with local only recurrence was 28.3 months (mean 36.1 (28.8); range 5.5-204.8). Median time to initial recurrence for these 74 patients was 16.8 months (mean 18.4 (12.0)). The 1 and 2 year post-recurrence survival rates were $48.7 \%$ and $17.6 \%$, respectively (fig 1). The sites of initial local recurrence and treatment for initial recurrence in these patients with local only recurrence are listed in table 2. Among the eight patients who underwent reoperation, five had stump recurrence while the other three patients had recurrence at the lung parenchyma. A stump recurrence was more likely to undergo resection. Characteristics and outcome of all 10 patients (eight with local only and two with local and distant recurrences) who underwent reoperation after local recurrence are available as an online supplement (see supplementary table 1 online). The initial pulmonary resection was lobectomy in five patients, bilobectomy in two, wedge resection in two and pneumonectomy in one. The operation for recurrence was completion pneumonectomy in four patients, lobectomy in three, wedge resection in two and stump revision in one. Two postoperative deaths occurred, both with completion pneumonectomy. The 5 year post-recurrence survival of the 10 patients was $15 \%$. Distant recurrences were detected in two patients after reoperation

Univariate analyses indicated that tumour size $(p=0.033)$ and treatment for initial recurrence $(p<0.001)$ had a significant influence on post-recurrence survival in 74 patients of resected stage I NSCLC with local only recurrence (table 3 ). The hazard of death was greater in patients with larger tumour size. Patients who received surgery as treatment for the initial

Table 2 Sites of initial local recurrence and treatment for initial recurrence in 74 patients with resected stage I non-small cell lung cancer with local only recurrence

\begin{tabular}{|c|c|c|c|c|c|}
\hline \multirow[b]{2}{*}{ Site of initial local recurrence } & \multirow[b]{2}{*}{$\begin{array}{l}\text { No of } \\
\text { patients }(\%)\end{array}$} & \multicolumn{4}{|c|}{ Treatment for initial recurrence } \\
\hline & & None & $\begin{array}{l}\text { With } \\
\text { surgery }\end{array}$ & $\begin{array}{l}\text { C/T and/or } \\
R / T\end{array}$ & Unknown \\
\hline Without mediastinal recurrence & $38(51.4)$ & & & & \\
\hline Stump & $16(21.6)$ & 0 & 5 & 7 & 4 \\
\hline Lung & $18(24.3)$ & 5 & 3 & 8 & 2 \\
\hline With pleural effusion & $4(5.5)$ & 0 & 0 & 2 & 2 \\
\hline With mediastinal recurrence & $18(24.3)$ & & & & \\
\hline Stump & $1(1.4)$ & 0 & 0 & 0 & 1 \\
\hline Lung & $3(4.1)$ & 1 & 0 & 2 & 0 \\
\hline With pleural effusion & $4(5.5)$ & 0 & 0 & 2 & 2 \\
\hline Mediastinal recurrence only & $10(13.3)$ & 1 & 0 & 4 & 5 \\
\hline Not specified & $18(24.3)$ & 0 & 0 & 1 & 17 \\
\hline
\end{tabular}

$\mathrm{C} / \mathrm{T}$, chemotherapy; $\mathrm{R} / \mathrm{T}$, radiotherapy. 
Table 3 Univariate analyses for post-recurrence survival in 74 patients with resected stage I non-small cell lung cancer with local only recurrence

\begin{tabular}{llr}
\hline Variable & Hazard ratio (95\% Cl) & p Value \\
\hline Age at recurrence (years) & $1.007(0.984$ to 1.031$)$ & 0.531 \\
Gender (male vs female) & $1.519(0.724$ to 3.184$)$ & 0.269 \\
Smoking index (pack-years) & $1.001(0.991$ to 1.010$)$ & 0.971 \\
Location (right vs left lung) & $0.697(0.419$ to 1.159$)$ & 0.697 \\
Tumour size (cm)* & $1.132(1.010$ to 1.267$)$ & 0.033 \\
Histological type (squamous cell carcinoma vs others) & $0.840(0.522$ to 1.351$)$ & 0.471 \\
Extent of pulmonary resection (sublobar resection vs lobectomy or & $1.644(0.813$ to 3.326$)$ & 0.167 \\
$\quad$ bilobectomy or pneumonectomy) & & \\
T status (T2 vs T1) & $1.220(0.746$ to 1.998$)$ & 0.428 \\
Visceral pleural invasion (present vs absent) & $0.932(0.524$ to 1.657$)$ & 0.811 \\
No of LNs dissected (>15 vs $\leqslant 15)$ & $1.101(0.644$ to 1.884$)$ & 0.725 \\
Disease free interval (months) $>18$ vs $\leqslant 18)$ & $0.921(0.573$ to 1.482$)$ & 0.736 \\
Mediastinal recurrence (present vs absent) & $1.031(0.969$ to 1.097$)$ & 0.335 \\
Treatment for initial recurrence & & $<0.001$ \\
$\quad$ None & 1 & \\
With surgery & $0.118(0.035$ to 0.400$)$ & \\
C/T and/or R/T & $0.319(0.134$ to 0.763$)$ & \\
\hline
\end{tabular}

*The hazard ratio associated with tumour size is that the increase in hazard is associated with a $1 \mathrm{~cm}$ increase in tumour size. $\mathrm{C} / \mathrm{T}$, chemotherapy; LN, lymph node; R/T, radiotherapy.

recurrence survived longer than those who received chemotherapy and/or radiotherapy. Furthermore, patients who received chemotherapy and/or radiotherapy survived longer than those who received no treatment. Treatment for initial recurrence $(p=0.001)$ was still a significant prognostic indicator in multivariate analyses (table 4). Patients with resected stage I NSCLC who underwent reoperation after local recurrence had a more favourable survival than those who received chemotherapy and/or radiotherapy and those who received no treatment. For the 38 patients with local only recurrence without mediastinal involvement, only treatment for the initial recurrence $(p=0.011)$ had a significant influence on post-recurrence survival in univariate analyses. Post-recurrence survival in patients with local only recurrence was not significantly different from that in those with both local and distant recurrences $(p=0.664)$.

\section{DISCUSSION}

Complete follow-up was achieved in 933 (96.2\%) of the 970 surgically resected stage I NSCLC patients from January 1980 to December 2000. The overall 5 year survival for all 933 patients was $51.7 \%$. Five year survival of surgically resected stage I NSCLC was reported as $55-80 \% .^{1-5}$ Our result is lower than in most other reports. Possible reasons include long period of patient collection, surgery performed by different surgeons and variations in mediastinal lymph node dissection. The number of mediastinal lymph nodes dissected represents the quality of lymphadenectomy in patients with resected stage I NSCLC. The quality of lymphadenectomy has impact on a more accurate tumour staging and affects the survival rate for patients with stage I NSCLC significantly. ${ }^{10}{ }^{11}$ The number of mediastinal lymph nodes dissected was 15 or less in $534(57.2 \%)$ and more than 15 in $382(41.0 \%)$ patients (unknown in 17 patients) in our study. For patients with more than 15 mediastinal lymph nodes dissected, 5 year survival reached $58.5 \%$.

This study investigated the prognostic role of conventional clinicopathological factors on post-recurrence survival in patients with resected stage I NSCLC with local recurrence. Local recurrence after surgical resection was detected in 123 $(13.2 \%)$ of the 933 patients. Median time to initial recurrence for these 123 patients was 15.2 months. The 1 and 2 year postrecurrence survival rates were $48.0 \%$ and $18.7 \%$, respectively. For 74 patients with local only recurrence, the 1 and 2 year postrecurrence survival rates were $48.7 \%$ and $17.6 \%$, respectively. Only treatment for initial recurrence was a significant predictor of post-recurrence survival in multivariate analyses.

Martini et al reported that the overall incidence of recurrence in patients with resected stage I NSCLC was $27 \%$ (local or regional $7 \%$, systemic $20 \%$ ). ${ }^{4}$ In the study of Harpole et al, the initial location of recurrence of stage I NSCLC after surgical resection was at a distant site in $19 \%$, within the ipsilateral hemithorax in $11 \%$ or at both locations in $6 \%$ of patients. ${ }^{5}$ Our results demonstrated that the overall incidence of recurrence was $31.0 \%$ (distant only in $17.8 \%$, local only in $7.9 \%$, local and distant in $5.3 \%)$. The patterns of local recurrence included local only in $74(60.2 \%)$, local with distant in $19(15.4 \%)$, local before distant in $24(19.5 \%)$ and distant before local in six (4.9\%) patients. Approximately $78 \%$ of patients with local recurrence occurred within the first 2 years after operation. Furthermore, our study followed the course and reoperation after local recurrence. More than half of the patients (51.3\%) died within 1 year after local only recurrence. The longest post-recurrence survival after local recurrence in our series was 15.8 years in a patient with stump recurrence who underwent reoperation.

Surgical resection offers a good chance of cure for patients with stage I NSCLC. ${ }^{1-5}$ However, the outcome of surgical treatment in resected stage I NSCLC after local recurrence has rarely been reported. Walsh et al reported that treatment with curative intent had significantly better overall survival and postrecurrence survival than treatment with palliative intent in

Table 4 Multivariate analyses for post-recurrence survival in 74 patients with resected stage I non-small cell lung cancer with local only recurrence

\begin{tabular}{lll}
\hline Variable & Hazard ratio $(95 \% \mathrm{Cl})$ & p Value \\
\hline Treatment for initial recurrence & & 0.001 \\
$\quad$ None & 1 & \\
With surgery & $0.089(0.024$ to 0.333$)$ & \\
$\mathrm{C} / \mathrm{T}$ and/or $\mathrm{R} / \mathrm{T}$ & $0.326(0.131$ to 0.813$)$ & \\
\hline
\end{tabular}

$\mathrm{C} / \mathrm{T}$, chemotherapy; $\mathrm{R} / \mathrm{T}$, radiotherapy. 
patients with NSCLC. ${ }^{16}$ Sugimura et al demonstrated that surgery or combination chemotherapy with radiation significantly improved post-recurrence survival over both no treatment and radiation alone in resected NSCLC after recurrence. ${ }^{7}$ Voltolini et al reported that 5 year survival after reoperation for locally recurrent bronchogenic carcinoma was $15.5 \%{ }^{8}$ The 5 year post-recurrence survival in our patients undergoing reoperation after local recurrence was 15\%. Our study further showed that surgical resection had a favourable survival in resected stage I NSCLC after local only recurrence (hazard ratio (HR) 0.089) than with chemotherapy and/or radiotherapy (HR $0.326)$ and with no treatment (HR 1.0, reference; $p=0.001$ ). Eight of 16 patients had no treatment for recurrence because of poor performance status. This may be a contributory factor to poor prognosis.

Recently, local and regional nodal recurrences were not considered the cause of significant cancer cell dissemination, distant metastases or death. ${ }^{1{ }^{18}}$ Rather, they are thought to be indicators of poor prognosis but not determinants of survival. Many studies have shown that better local control increases survival in some cancers. ${ }^{18-23}$ On the other hand, distant metastasis represents dissemination of cancer cells in distant organs and has significant impact on survival. ${ }^{124}$ Our study showed that patients who underwent complete resection for local only recurrence survived longer than those who received chemotherapy and/or radiotherapy and those who received no treatment. However, post-recurrence survival in patients with local only recurrence was not significantly different from those with both local and distant recurrences in our study. The seventh edition of the TNM Classification of Malignant Tumours is scheduled to be published in 2009. For lung cancer, some studies propose that pleural effusion be moved from T4 to M1a. ${ }^{25-27}$ Our results showed that post-recurrence survival in patients with pleural effusion as part of the local only recurrence was not significantly different from that in those without pleural effusion $(p=0.432)$.

Treatment for recurrence is a prognostic predictor for postrecurrence survival in resected stage I NSCLC with local recurrence. Complete surgical resection should be considered in selected candidates with resectable recurrent disease.

Acknowledgements: The authors are grateful to Drs Liang-Shun Wang of En Chu Kong Hospital and Wen-Juei Jeng of Chang Gung Memorial Hospital for their contribution to this article. They also thank Mr Jung-Hsing Lin for his assistance in data collection.

Competing interests: None.

\section{REFERENCES}

1. Non-Small Cell Lung Cancer Collaborative Group. Chemotherapy in non-small cell lung cancer: a meta-analysis using updated data on individual patients from 52 randomized clinical trials. Non-Small Cell Lung Cancer Collaborative Group. BMJ 1995:311:899-905.
2. Mountain CF. Revisions in the international system for staging lung cancer. Chest 1997:111:1710-17.

3. Nesbitt JC, Putnam JB Jr, Walsh GL, et al. Survival in early-stage non-small cell lung cancer. Ann Thorac Surg 1995;60:466-72.

4. Martini N, Bains MS, Burt ME, et al. Incidence of local recurrence and second primary tumors in resected stage I lung cancer. J Thorac Cardiovasc Surg 1995; 109:120-9

5. Harpole DH Jr, Herndon JE II, Young WG Jr, et al. Stage I non-small cell lung cancer. Cancer 1995;76:787-96.

6. Al-Kattan K, Sepsas E, Fountain SW, et al. Disease recurrence after resection for stage I lung cancer. Eur J Cardiothorac Surg 1997;12:380-4.

7. Sugimura $\mathbf{H}$, Nichols FC, Yang $\mathrm{P}$, et al. Survival after recurrent nonsmall-cell lung cancer after complete pulmonary resection. Ann Thorac Surg 2007;83:409-18.

8. Voltolini L, Paladini P, Luzzi L, et al. Iterative surgical resections for local recurrent and second primary bronchogenic carcinoma. Eur J Cardiothorac Surg 2000;18:529-34.

9. Williams BA, Sugimura H, Endo C, et al. Predicting postrecurrence survival among completely resected nonsmall-cell lung cancer patients. Ann Thorac Surg 2006;81:1021-7.

10. Wu YC, Lin CF, Hsu WH, et al. Long-term results of pathological stage I non-small cell lung cancer: validation of using the number of totally removed lymph nodes as a staging control. Eur J Cardiothorac Surg 2003;24:994-1001.

11. Hung JJ, Wang CY, Huang MH, et al. Prognostic factors in resected stage I nonsmall cell lung cancer with a diameter of $3 \mathrm{~cm}$ or less: visceral pleural invasion did not influence overall and disease-free survival. $J$ Thorac Cardiovasc Surg 2007:134:638-43

12. World Health Organization. Histological typing of lung tumors, 2nd Edn. Geneva: World Health Organization, 1981.

13. Sobin LH, Wittekind C. International Union Against Cancer: TNM classification of malignant tumours, 5th Edn. New York: Wiley-Liss, 1997.

14. Martini N, Melamed MR. Multiple primary lung cancers. J Thorac Cardiovasc Surg 1975:70:606-12.

15. Kaplan EL, Meier P. Nonparametric estimation for incomplete observations. J Am Stat Assoc 1958;53:457-81.

16. Walsh GL, O'Connor M, Willis KM, et al. Is follow-up of lung cancer patients after resection medically indicated and cost-effective? Ann Thorac Surg 1995;60:1563-70.

17. Early Breast Cancer Trialists' Collaborative Group. Effects of radiotherapy and surgery in early breast cancer. An overview of the randomized trials. N Engl J Med 1995; 333:1444-55.

18. Cady B. Fundamentals of contemporary surgical oncology: biologic principles and the threshold concept govern treatment and outcomes. J Am Coll Surg 2001;192:777-92.

19. Overgaard M, Hansen PS, Overgaard J, et al. Postoperative radiotherapy in high-risk premenopausal women with breast cancer who receive adjuvant chemotherapy. N Engl J Med 1997;337:949-55

20. Ragaz J, Jackson SM, Le N, et al. Adjuvant radiotherapy and chemotherapy in nodepositive premenopausal women with breast cancer. N Engl J Med 1997;337:956-62.

21. Whelan TJ, Julian $\mathrm{J}$, Wright $\mathrm{J}$, et al. Does locoregional radiation therapy improve survival in breast cancer? A meta-analysis. J Clin Oncol 2000;18:1220-9.

22. Kattan MW, Zelefsky MJ, Kupelian PA, et al. Pretreatment nomogram for predicting the outcome of three-dimensional conformal radiotherapy in prostate cancer. J Clin Oncol 2000;18:3352-9.

23. Cohen AM. Radical surgery for rectal cancer: why we fail and rationale for current clinical trials of adjuvant therapy. Surg Oncol Clin N Am 2000;9:741-7.

24. Chambers AF, Naumov GN, Varghese HJ, et al. Critical steps in hematogenous metastasis: an overview. Surg Oncol Clin N Am 2001;10:243-55.

25. Postmus PE, Brambilla E, Chansky K, et al. The IASLC Lung Cancer Staging Project: proposals for revision of the $\mathrm{M}$ descriptors in the forthcoming (seventh) edition of the TNM classification of lung cancer. J Thorac Oncol 2007;2:686-93.

26. Groome PA, Bolejack V, Crowley JJ, et al. The IASLC Lung Cancer Staging Project: validation of the proposals for revision of the $\mathrm{T}, \mathrm{N}$, and $\mathrm{M}$ descriptors and consequent stage groupings in the forthcoming (seventh) edition of the TNM classification of malignant tumours. J Thorac Oncol 2007;2:694-705.

27. Goldstraw P, Crowley J, Chansky K, et al. The IASLC Lung Cancer Staging Project: proposals for the revision of the TNM stage groupings in the forthcoming (seventh) edition of the TNM Classification of malignant tumours. J Thorac Oncol 2007;2:706-14. 